\title{
Editorial: special issue on advances in security and privacy for future mobile communications
}

\author{
Georgios Kambourakis ${ }^{1}$ - Gregorio Martínez ${ }^{2}$. \\ Félix Gómez Mármol ${ }^{3}$
}

Recent advances in wireless communication technologies along with a proliferation of mobile devices have enabled a new, and expanded level of ubiquitous access to data and communications. Reliable, secure, and private communications are as essential in these product offerings as network availability, especially as the Internet presents enhanced opportunities for network breaches. Every day new security challenges surface, often a consequence of weak encryption and inherently open access to networks and in some cases lack of a pre-deployed infrastructure. This special issue advances the state-of-the-art research in the area of security, privacy, cryptography, and its applications to future mobile communications. Our special issue emphasizes potential contributions to security and privacy preserving applications and services in the mobile ecosystem. It contains seven papers, in print and online release, covering anonymous RA for trusted computing, cooperative security system for m-Health applications, security solutions for the IMS media plane, a comprehensive survey on Anonymous Voice over IP (VoIP) communication, and an untraceable authentication method for large-scale active-tag RFID systems. These papers will hopefully stimulate further research in this important topic.

Georgios Kambourakis

gkamb@aegean.gr

1 University of the Aegean, Samos, Greece

2 University of Murcia, Murcia, Spain

3 Security group at NEC Laboratories Europe, Heidelberg, Germany 
Georgios Kambourakis is assistant professor in the Department of Information and Communication Systems Engineering of the University of the Aegean. His research interests are in the fields of mobile and wireless networks security and privacy, VoIP security, PKI, DNS security, and mLearning and he has more than 100 publications in the above areas.

Gregorio Martínez is full professor in the Department of Information and Communications Engineering of the University of Murcia. He has published more than a hundred journal articles and conference papers and has been involved as collaborator or supervisor in several open-source software projects.

Félix Gómez Mármol senior researcher in the security group at NEC Laboratories Europe, Heidelberg, Germany. He published over 30 research articles, holds four patents and participated in several special issues as guest editor in international journals. 